\title{
Understanding Depletion Forces beyond Entropy
}

\author{
C. Bechinger, D. Rudhardt, and P. Leiderer \\ Fakultät für Physik, Universität Konstanz, D-78457 Konstanz, Germany \\ R. Roth and S. Dietrich \\ Fachbereich Physik, Bergische Universität Wuppertal, D-42097 Wuppertal, Germany
}

(Received 23 August 1999)

\begin{abstract}
The effective interaction energy of a colloidal sphere in a suspension containing small amounts of nonionic polymers and a flat glass surface has been measured and calculated using total internal reflection microscopy and a novel approach within density functional theory, respectively. Quantitative agreement between experiment and theory demonstrates that the resulting repulsive part of the depletion forces cannot be interpreted entirely in terms of entropic arguments but that particularly at small distances $(\$ 100 \mathrm{~nm})$ attractive dispersion forces have to be taken into account.
\end{abstract}

PACS numbers: 82.70.Dd, 36.20.-r

The stability of systems like polydisperse mixtures of colloids, natural rubbers, micelles, or polymer coils is known to be strongly influenced by depletion forces. In addition, such forces may also play an important role in biological systems, e.g., in promoting the aggregation of red blood cells $[1,2]$. The understanding of these forces being responsible for spontaneous structure formation, phase separation, or flocculation in such systems is highly demanding both from the experimental and the theoretical points of view. A first approximate explanation of depletion forces was given by Asakura and Oosawa (AO), who recognized that the presence of small hard spheres (referred to as macromolecules in the following) can mediate effective forces between two larger objects if their distance is sufficiently small [3]. This can be easily understood by considering, e.g., a hard sphere of radius $R$ suspended in a hard-sphere fluid of macromolecules with radius $r$ and bulk number density $\rho_{b}$ in front of a wall (or another big sphere) at distance $z$, measured between the surfaces of the wall and the spheres. For $z<2 r$, the macromolecules are expelled from a region of excluded volume overlap, i.e., their density is depleted at that hemisphere of the large particle facing the wall compared to the opposite side which faces the bulk liquid. Such density gradients give rise to an effective osmotic pressure, causing the big sphere to be pushed towards the wall thereby allowing the entropy of the macromolecules to increase. Accordingly, these depletion forces result from an asymmetric density distribution of the macromolecules around the big sphere. Quantitative calculations of depletion forces require the knowledge of the density distribution $\rho(\mathbf{r})$ of the macromolecules. Within the crudest approximation $\rho(\mathbf{r})$ is considered to be constant (corresponding to the above mentioned AO approximation), so that depletion forces can be easily calculated in terms of excluded volume arguments and are predicted to be entirely attractive for $z<2 r$. For sufficiently low macromolecule densities this approximation is in agreement with experimental results $[4,5]$. If, however, at high concen- trations, the structural correlation effects in the macromolecular liquid become important, $\rho(\mathbf{r})$ displays an oscillatory behavior at small values of $z$ generating repulsive parts of the depletion interactions as observed recently [6]. By means of virial coefficient expansions within the Derjaguin approximation [7] and by other techniques in [8] it has been found that the repulsive interaction strength strongly increases for large values of $R / r$.

So far the interpretation of the corresponding experiments has been based on assuming hard-sphere macromolecule and hard-wall interactions which is, however, not always justified under experimental conditions. This is most evident in the case of highly charged macromolecules where even at volume fractions $\rho_{b} 4 \pi r^{3} / 3 \lesssim 0.02$ electrostatic interactions lead to long-range repulsive and shortrange attractive parts in the depletion potential between a sphere and the wall $[9,10]$. But even for systems of uncharged macromolecules or in the high salt limit, the model of hard spheres and a hard wall is not applicable within the range of attractive dispersion forces.

Here we present measurements of the depletion potential of a single colloidal polystyrene (PS) sphere in front of a flat glass surface in the presence of nonionic macromolecules for various values of the macromolecule density $\rho_{b}$. In addition we have calculated the depletion potential within density functional theory (DFT) for a hardsphere system in which the dispersion and electrostatic interactions between the macromolecules and the wall have been taken into account. We find clear evidence for the occurrence of attractive and repulsive interaction energies both in our experiment and in our calculations. The latter demonstrate that dispersion forces considerably modify the depletion potential below $100 \mathrm{~nm}(\simeq r)$. This substantiates the hypothesis [11] that in general for depletion forces both entropic and energetic contributions are important.

We have used total internal reflection microscopy (TIRM) [12] to measure the potential energy of a colloidal sphere close to a glass surface. In this technique, a laser beam is reflected from a solid-fluid interface slightly above 
the critical angle of total reflection thus generating an evanescent wave in the fluid. A colloidal sphere located sufficiently close to the interface scatters the evanescent wave with an intensity which decays exponentially with increasing particle-interface distance. Thus the scattered intensity, which fluctuates owing to Brownian motion, determines sensitively and instantaneously the separation distance $z$. In order to obtain the spatial dependence of the potential energy of the particle one has to measure the separation distances sampled by the colloidal sphere for a statistically long period of time. From this, the probability of finding the particle at any separation distance can be calculated which is related to the potential energy via the Boltzmann distribution. If the penetration depth of the evanescent wave is chosen to be of the order of several hundred nanometers, this technique allows one to determine precisely effective particle-wall interaction potentials close to an interface.

Monodisperse PS particles of radius $R=5 \mu \mathrm{m}$ were stabilized with sulfate surface groups which dissociate in contact with water and thus cause the particles to be negatively charged. Owing to the large weight of the PS spheres, it was necessary to increase their buoyancy to facilitate large enough thermally driven vertical excursions of the particles during the measurements. Therefore the particles were suspended in a mixture of deuterated water and normal water which caused a reduction of the weight by a factor of 8.5. As macromolecules we chose poly(ethylene oxide) (PEO) which had been successfully employed in other studies of depletion effects before $[4,5,13]$. PEO is a nonionic water-soluble polymer which forms polymer coils below a critical density $\rho_{c}$. For the molecular weight which was used in our experiments $\left(M_{W}=10^{6}\right)$ the radius of gyration $r_{G}$ has been determined by means of static light scattering to be $r_{G}=67.7 \mathrm{~nm}$ [14]. The maximum polymer number density used in our experiments was $23.4 \mu \mathrm{m}^{-3}$ which is smaller than $\rho_{c}$ by more than a factor of 30 . Accordingly in the present context the polymer coils can be considered as spherical macromolecules to be modeled in our calculations as hard spheres $\left(\rho_{b} \ll \rho_{c}\right)$ with an effective radius $r=107 \mathrm{~nm}$ [15].

The sample cell was composed of two silica flats separated by a $1 \mathrm{~mm}$ thick O-ring and was connected to a closed circuit system containing an ion exchange resin and a conductivity probe. This setup allowed us to control precisely the ionic strength of the suspension. The evanescent field was generated by a HeNe-laser beam $(\lambda=633 \mathrm{~nm}$, $P=5 \mathrm{~mW}$ ) which was coupled to the bottom plate of the cell under total internal reflection conditions by means of a glass prism. The penetration depth was chosen to be $400 \mathrm{~nm}$. Highly diluted suspensions with PS number densities below $0.5 \mathrm{~mm}^{-3}$ have been used to ensure that only a single PS particle was in the field of view and contributed to the scattering signal. The scattered intensity of the PS sphere was collected by a microscope objective and focused onto a photomultiplier tube. In order to obtain suf- ficient data and to minimize statistical errors the scattered intensity of a particle was sampled over at least $1000 \mathrm{~s}$ at a sampling rate of $50 \mathrm{~Hz}$.

In the absence of depletion forces, i.e., without adding macromolecules, the potential of a negatively charged PS particle above a glass plate is composed of two parts. Towards smaller distances the potential increases exponentially owing to the electrostatic interaction between the sphere and the fused silica plate, which is also negatively charged when in contact with water. For larger distances the potential is completely dominated by gravity. It has been shown theoretically and experimentally that under these conditions the external potential is given by [12]

$$
\beta \Phi_{\mathrm{ext}}^{\mathrm{PS}}(z)=B \exp (-\kappa z)+\beta G z,
$$

where $B$ depends on the sphere and the glass plate surface potentials, $\kappa$ is the inverse Debye screening length related to the ionic strength of the suspension, and $\beta=\left(k_{B} T\right)^{-1}$. $G=\left(\bar{\rho}_{\text {PS }}-\bar{\rho}_{W}\right) V g$ is the weight of the particle of volume $V$ suspended in water, $\bar{\rho}_{\text {PS }}$ and $\bar{\rho}_{W}$ are the mass densities of PS and water, respectively, and $g$ is the acceleration of gravity. The values for $V$ and $\bar{\rho}_{\text {PS }}$ were provided by the manufacturer.

This potential is shown in Fig. 1(a) as measured ( $\square$ ). The PS sphere probes a range of about $300 \mathrm{~nm}$ from the surface during our measuring time. Because of the large weight of the PS sphere with $R=5 \mu \mathrm{m}$, we are very sensitive to changes in the sphere-wall potential below $z=150 \mathrm{~nm}$; for smaller spheres this potential is dominated by the electrostatic repulsion [5]. The solid line in Fig. 1 $(a)$ is the fit curve according to Eq. (1) with the two fit parameters $B=4.81$ and $\kappa^{-1}=17 \mathrm{~nm}$ (for $T=$ $298 \mathrm{~K}$ ), the latter being in agreement with the electrical conductivity measurement of the colloidal circuit. This demonstrates that in Eq. (1) attractive dispersion forces are negligible.

However, for the macromolecules dispersion and electrostatic interactions between them and the glass plate have to be taken into account. The latter originate from the fact that the charged glass plate induces a dipole moment in the neutral macromolecules which interacts with the electric field resulting in a repulsion due to the much smaller static index of refraction $\varepsilon_{1}$ of PEO than that of water $(\varepsilon=81)$. This leads to

$$
\Phi_{\mathrm{ext}}^{\mathrm{PEO}}(z)=C \exp [-2 \kappa(z+r)]-A\left(\frac{r}{z+r}\right)^{3}
$$

with the Hamaker constant $A=5 \times 10^{-20} \mathrm{~J}[16]$ and

$$
C=12 \pi[\kappa r \cosh (\kappa r)-\sinh (\kappa r)] \frac{\varepsilon \varepsilon_{0}\left(\varepsilon-\varepsilon_{1}\right)}{\varepsilon_{1}+2 \varepsilon} \frac{E_{0}^{2}}{\kappa^{3}}
$$

with $\varepsilon_{1} \approx 5$ [17], the surface electric potential of the glass plate $E_{0} \approx 50 \mathrm{mV}$ [18], and $\varepsilon_{0}$ the permittivity of vacuum. Estimates indicate that for the densities $\rho_{b}$ considered here the attractive dispersion forces among the macromolecules and between them and the PS spheres are not important. 


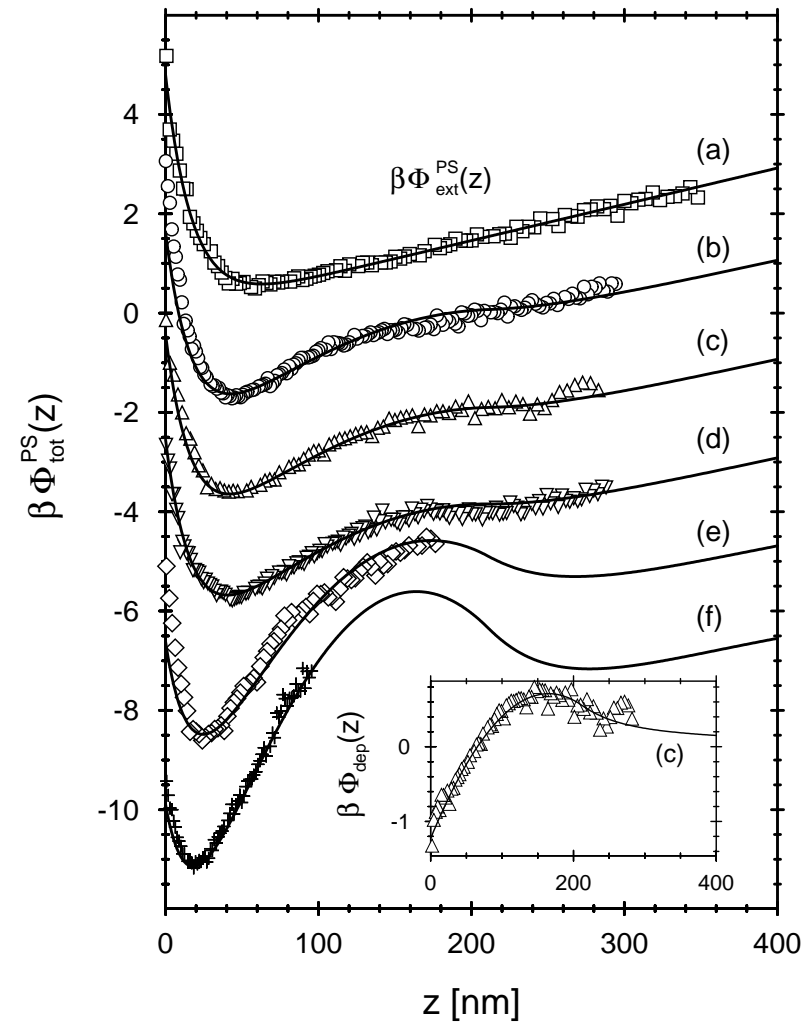

FIG. 1. Comparison of the effective potential $\Phi_{\text {tot }}^{\mathrm{PS}}(z)=$ $\Phi_{\mathrm{ext}}^{\mathrm{PS}}(z)+\Phi_{\mathrm{dep}}(z)$ acting on the big PS particle between theory (solid lines) and experiment (symbols) for various number densities of the macromolecules: (a) $\rho_{b}^{\exp }=0$, (b) $4.1 \mu \mathrm{m}^{-3}$, (c) $6.3 \mu \mathrm{m}^{-3}$, (d) $8.7 \mu \mathrm{m}^{-3}$, (e) $16.4 \mu \mathrm{m}^{-3}$, and $(f) 23.4 \mu \mathrm{m}^{-3}$. For reasons of clarity the curves have been separated from each other vertically by 4 units.

The agreement, which we find between our experimental data and our calculations without considering the latter forces, supports this approximation a posteriori.

When PEO is added to the system the presence of depletion forces generates modified effective potentials acting on the PS sphere. Figure 1 shows the measured potentials (symbols) $\Phi_{\mathrm{tot}}^{\mathrm{PS}}(z)=\Phi_{\mathrm{ext}}^{\mathrm{PS}}(z)+\Phi_{\mathrm{dep}}(z)$ when different amounts of PEO are added to the suspension. Assuming a homogeneous distribution of PEO over the whole circuit, from the experimentally determined weights, the PEO number densities are obtained as $(b) \rho_{b}^{\text {exp }}=$ $4.1 \mu \mathrm{m}^{-3}$, (c) $6.3 \mu \mathrm{m}^{-3}$, (d) $8.7 \mu \mathrm{m}^{-3}$, (e) $16.4 \mu \mathrm{m}^{-3}$, and $(f) 23.4 \mu \mathrm{m}^{-3}$, respectively. With increasing PEO concentration the potential deepens and the position of the minimum considerably shifts to smaller values of $z$ in agreement with previous measurements [5]. Additionally, however, we find a bump in the potential around $z=$ $150 \mathrm{~nm}$ which increases with increasing PEO densities. In order to highlight this feature we have subtracted the electrostatic and gravitational contribution [Eq. (1)] from Fig. $1(c)$ and present this difference in the inset of Fig. 1 as measured $(\triangle)$ and as calculated (solid line). Within $\Phi_{\text {dep }}$ the bump turns into a pronounced maximum with a height of approximately $0.75 k_{B} T$ which gives rise to a repulsive depletion force of the PS sphere upon approaching the wall before it finally becomes attractive for $z$ smaller than $150 \mathrm{~nm}$.

Our theoretical calculations for $\Phi_{\text {tot }}^{\mathrm{PS}}(z)$ are based on a novel and versatile DFT approach [19]. It has been shown that in the limit of present interest, i.e., when the density of PS goes to zero, the depletion potential $\Phi_{\text {dep }}(z)$ can be determined in two steps solely from the density profile of the macromolecules $\rho_{\mathrm{PEO}}(z)$ without PS sphere and from the geometrical shape of the PS particle, i.e., its radius $R$ [19]. First, the density profiles of the polymers $\rho_{\mathrm{PEO}}(z)$ subjected to the external potential given by Eq. (2) have been obtained by freely minimizing the Rosenfeld functional [20]. With all parameters in the external potential and the effective hard-sphere radius $r$ of the macromolecules fixed, the only adjustable parameter in the calculation is the PEO bulk density. In Fig. 2 we show a PEO density profile for $\rho_{b}=4.1 \mu \mathrm{m}^{-3}$ (solid line). The density profile reflects the short-ranged electrostatic repulsion at very small distances and the dispersion attraction leading to a pronounced maximum more than $50 \%$ above the bulk value. The importance of $\Phi_{\mathrm{ext}}^{\mathrm{PEO}}$ for bringing about this structure is underscored by the dotted line in Fig. 2 which corresponds to $\Phi_{\mathrm{ext}}^{\mathrm{PEO}} \equiv 0$. Second, from such PEO density profiles we have calculated the effective potentials shown as solid lines in Figs. 1 $(b)-1(f)$. As mentioned above, the only adjustable parameter in the calculations is $\rho_{b}$ which has been varied until the best agreement with the experimental data has been obtained leading to the theoretical values $(b) \rho_{b}^{\text {th }}=3.9 \mu \mathrm{m}^{-3}$, (c) $4.1 \mu \mathrm{m}^{-3}$, (d) $4.5 \mu \mathrm{m}^{-3}$, (e) $11.3 \mu \mathrm{m}^{-3}$, and $(f) 15.5 \mu \mathrm{m}^{-3}$, respectively. We find very good agreement with the experimental potential data and, in particular, the emerging barrier for increasing $\rho_{b}$

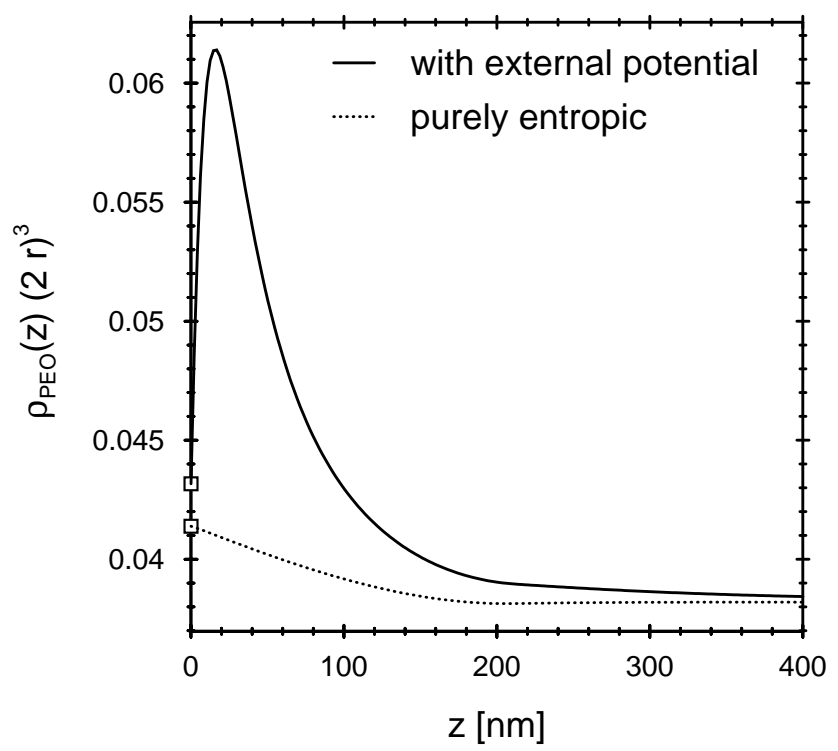

FIG. 2. The number density profile $\rho_{\mathrm{PEO}}(z)$ of the macromolecules in the absence of a PS sphere for the wall potential $\Phi_{\mathrm{ext}}^{\mathrm{PEO}}(z)$ given by Eq. (2) (solid line) and for a hard wall (dotted line); the theoretical value $\rho_{b}^{\text {th }}=4.1 \mu \mathrm{m}^{-3}$ corresponds to Fig. $1(c)$ and $\rho_{\mathrm{PEO}}(z=0)(2 r)^{3}=0.043$. 


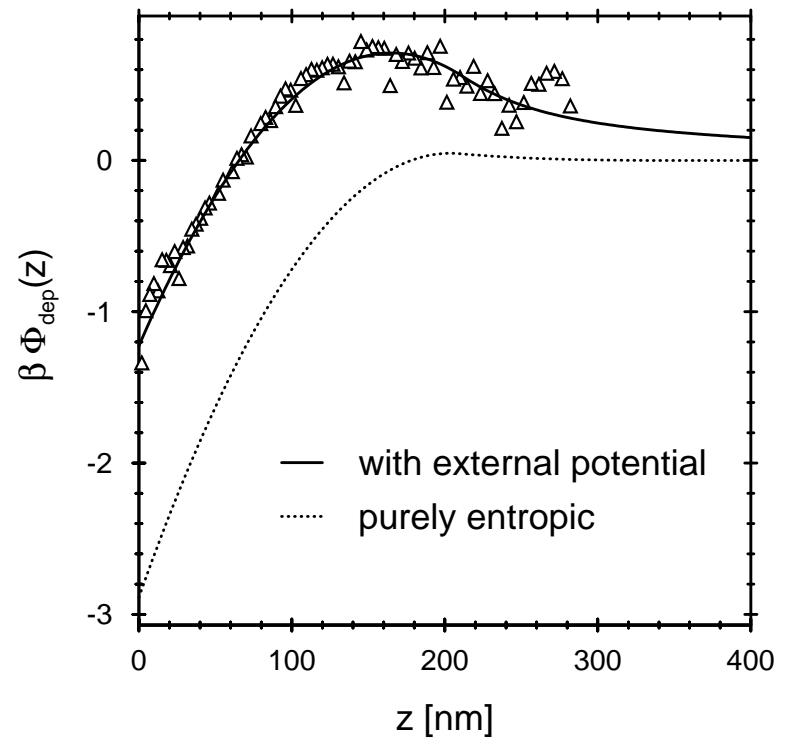

FIG. 3. The depletion potentials $\Phi_{\text {dep }}(z)$ between the wall and a big PS sphere calculated from the corresponding PEO density profiles $\rho_{\mathrm{PEO}}(z)$ shown in Fig. 2 . $\triangle$ denotes the corresponding experimental data.

is reproduced. The values of $\rho_{b}^{\text {th }}$ agree within a factor of about 1.5 with $\rho_{b}^{\exp }$. A possible reason for these differences could be due to lateral density gradients within the experimental circuit not captured by the aforementioned global determination of $\rho_{b}^{\exp }$.

We emphasize that the potential barriers in Fig. 1 cannot be explained in terms of a hard-sphere and hard-wall model, i.e., by purely entropic arguments. This conclusion is demonstrated in Fig. 3 by the discrepancy between the actual depletion potential and the one close to a hard wall.

Another important conclusion which can be drawn from our calculations is that, although the dispersion attraction seems to be mainly responsible for the potential barrier, the soft electrostatic repulsion plays an important role as well. By changing the salt concentration and hence the inverse screening length, one can experimentally tune the range of the electrostatic repulsion and thus modify the potential barrier. This explains why in an earlier experiment with a lower salt concentration no repulsive depletion forces were found [5].

So far, we have demonstrated that DFT allows one to calculate accurately the depletion force on a suspended sphere from the density profile of the dissolved macromolecules in the absence of the big sphere. For small macromolecule densities this relationship can be inverted: measuring the depletion potential of a large sphere may be used to determine the undisturbed macromolecule density profile. This is a remarkable result, because the big sphere considerably modifies the density distribution of macromolecules. Thus depletion force measurements can be used as a tool for determining undisturbed solvent properties.

In summary, we have studied the depletion potential of a single polystyrene sphere in a fluid of nonionic macro- molecules in front of a wall. The potential exhibits both attractive as well as repulsive parts which are not of purely entropic origin but are largely dominated by the dispersion forces between the uncharged macromolecules and the wall. These results are in good quantitative agreement with density functional theory calculations carried out specificly for this system.

C.B., D. R., and P.L. acknowledge financial support from the Deutsche Forschungsgemeinschaft through SFB 513.

[1] K. Mayer, E. Hahnel, and R. R. Feiner, Proc. Roy. Exp. Biol. Med. 58, 36 (1945).

[2] J. Janzen and D. E. Brooks, Clinical Hemorheology 9, 695 (1989).

[3] S. Asakura and F. Oosawa, J. Chem. Phys. 22, 1255 (1954).

[4] Y. N. Ohshima et al., Phys. Rev. Lett. 78, 3963 (1997).

[5] D. Rudhardt, C. Bechinger, and P. Leiderer, Phys. Rev. Lett. 81, 1330 (1998).

[6] J.C. Crocker, J.A. Matteo, A.D. Dinsmore, and A. G. Yodh, Phys. Rev. Lett. 82, 43521999.

[7] Y. Mao, M.E. Cates, and H. N. W. Lekkerkerker, Physica (Amsterdam) 222A, 10 (1995).

[8] B. Götzelmann, R. Evans, and S. Dietrich, Phys. Rev. E 57, 6785 (1998).

[9] D. L. Sober and J. Y. Walz, Langmuir 11, 2352 (1995).

[10] A. Sharma and J. Y. Walz, J. Chem. Soc. Faraday Trans. 92, 4997 (1996).

[11] A.D. Dinsmore and A.G. Yodh, Langmuir 15, 314 (1999).

[12] D. C. Prieve, F. Luo, and F. Lanni, Faraday Discuss. Chem. Soc. 83, 297 (1987); D. Haughey and J.C. Earnshaw, Colloids Surf. A 136, 217 (1998).

[13] D. Rudhardt, C. Bechinger, and P. Leiderer, Prog. Colloid Polym. Sci. 112, 163 (1999).

[14] K. Devanand and J. C. Selser, Macromolecules 24, 5943 (1991).

[15] If due to thermal averaging PEO is considered as a homogeneous hard sphere with radius $r$, this value is obtained from the radius of gyration by $r=\sqrt{5 / 2} r_{G}$ which yields $r=107$ for $r_{G}=67.7 \mathrm{~nm}$. Such a relationship between $r_{G}$ and $r$ is also in agreement with other experiments [4,5].

[16] J. Israelachvili, Intermolecular \& Surface Forces (Academic, London, 1992).

[17] No accurate values for $\varepsilon_{1}$ were found in the literature. Therefore we varied $\varepsilon_{1}$ between 3 and 10 which did not result in any visible changes of either the density profiles or the depletion potentials.

[18] S. Tanimoto, H. Matsuoka, H. Yamauchi, and H. Yamaoka, Colloid Polym. Sci. 277, 130 (1999).

[19] B. Götzelmann, R. Roth, S. Dietrich, M. Dijkstra, and R. Evans, Europhys. Lett. 47, 398 (1999).

[20] Y. Rosenfeld, Phys. Rev. Lett. 63, 980 (1989). 